PROCEEDINGS OF THE

AMERICAN MATHEMATICAL SOCIETY

Volume 137, Number 4, April 2009, Pages 1357-1361

S 0002-9939(08)09673-1

Article electronically published on October 6, 2008

\title{
INVARIANT SUBSPACES OF SUPER LEFT-COMMUTANTS
}

\author{
HAILEGEBRIEL E. GESSESSE
}

(Communicated by Nigel J. Kalton)

\begin{abstract}
For a positive operator $Q$ on a Banach lattice, one defines $\langle Q]=$ $\{T \geq 0: T Q \leq Q T\}$ and $[Q\rangle=\{T \geq 0: T Q \geq Q T\}$. There have been several recent results asserting that, under certain assumptions on $Q,[Q\rangle$ has a common invariant subspace. In this paper, we use the technique of minimal vectors to establish similar results for $\langle Q]$.
\end{abstract}

Throughout this paper, we assume that $X$ is a real Banach lattice with positive cone $X_{+} ; \mathcal{L}(X)$ stands for the space of all (bounded linear) operators on $X$. Let $Q$ be a positive operator on $X$. By an invariant subspace of $Q$ we mean a closed subspace $V$ of $X$ such that $V \neq\{0\}, V \neq X$ and $Q V \subseteq V$. The super left-commutant $\langle Q]$ and the super right-commutant of $[Q\rangle$ of $Q$ are defined as follows:

$$
\langle Q]=\{T \geq 0: T Q \leq Q T\}, \quad[Q\rangle=\{T \geq 0: T Q \geq Q T\} .
$$

The symbol $B(x, r)$ stands for the closed ball of radius $r$ centered at $x$. If $a<b$ in $X$, we write $[a, b]=\{x \in X: a \leq x \leq b\}$. A subspace $Y \subseteq X$ is an (order) ideal if $|y| \leq|x|$ and $x \in Y$ imply $y \in Y$. For $K \in \mathcal{L}(X)$ we say that $K$ is dominated by $Q$ if $|K x| \leq Q|x|$ for every $x \in X$. Obviously, every operator in $[0, Q]=\{K \in \mathcal{L}(X): 0 \leq K \leq Q\}$ is dominated by $Q$. For more details on positive operators, we refer the reader to [AA02].

Suppose that $Q$ is compact-friendly (see the definition below) and quasinilpotent. It was shown in AA02 that every sequence in $[Q\rangle$ has a (common) invariant subspace, which is also invariant under $Q$. Furthermore, if $X$ is order complete, then the entire $[Q\rangle$ has an invariant subspace. Using the technique of minimal vectors (see [AE98, Tr04, AT05, GT]) we prove in this paper that the same results hold for $\langle Q]$. First we prove an extension of a fact in AT05.

Definition 1. A collection of operators $\mathcal{F} \subseteq \mathcal{L}(X)$ localizes a set $A \subseteq X$ if for every sequence $\left(x_{n}\right)$ in $A$ there exists a subsequence $\left(x_{n_{i}}\right)$ and a sequence $\left(K_{i}\right)$ in $\mathcal{F}$ such that $K_{i} x_{n_{i}}$ converges to a non-zero vector.

Theorem 2 ([AT05]). Suppose that $Q$ is a positive quasinilpotent one-to-one operator with dense range and $x_{0} \in X_{+}$with $\left\|x_{0}\right\|>1$. If the set of all operators dominated by $Q$ localizes $B\left(x_{0}, 1\right) \cap\left[0, x_{0}\right]$, then there exists an invariant subspace for $\langle Q]$. Moreover, if $[0, Q]$ localizes $B\left(x_{0}, 1\right) \cap\left[0, x_{0}\right]$, then $\langle Q]$ has an invariant closed ideal.

Received by the editors April 11, 2008

2000 Mathematics Subject Classification. Primary 47A15; Secondary 46B42, 47B65.

Key words and phrases. Invariant subspace, quasinilpotent operator, positive operator.

(C)2008 American Mathematical Society Reverts to public domain 28 years from publication 
We extend Theorem 2 as follows.

Theorem 3. Suppose that $Q$ is a positive quasinilpotent operator and $x_{0} \in X_{+}$with $\left\|x_{0}\right\|>1$. If there exists $R$ in $\langle Q]$ such that the set of all operators dominated by $R$ localizes $B\left(x_{0}, 1\right) \cap\left[0, x_{0}\right]$, then there exists an invariant subspace for $\langle Q]$. Moreover, if $[0, R]$ localizes $B\left(x_{0}, 1\right) \cap\left[0, x_{0}\right]$, then $\langle Q]$ has an invariant closed ideal.

Proof. Suppose $R \in\langle Q]$ such that the set of all operators dominated by $R$ localizes $B\left(x_{0}, 1\right) \cap\left[0, x_{0}\right]$. Since the ideal generated by Range $Q$ is invariant under $\langle Q]$ by GT, Lemma 0.5], we assume that this ideal is dense in $X$. As in the proof of [AT05, Theorem 8] and [GT, Theorem 5.5], we find a sequence $\left(K_{i}\right)$ of operators dominated by $R$ and an increasing sequence of integers $\left(n_{i}\right)$ such that $K_{i}\left(x_{0} \wedge Q^{n_{i}-1} y_{n_{i}-1}\right)$ converges to some vector $w \neq 0$, and $\left(f_{n_{i}}\right) \mathrm{w}^{*}$-converges to a positive functional $g \neq 0$, where $\left(y_{n}\right)$ is a sequence of 2-minimal vectors and $\left(f_{n}\right)$ is a sequence of 2-minimal functionals for $Q$ and $B\left(x_{0}, 1\right)+X_{+}$.

Suppose $T \in\langle Q]$. Using the facts that $K_{i}$ is dominated by $R$ for each $i, T Q \leq$ $Q T, R Q \leq Q R$, and by Propositions 5.3(v) and 5.4 of [GT], we have

$$
\begin{aligned}
& \left|f_{n_{i}}\left(Q T K_{i}\left(x_{0} \wedge Q^{n_{i}-1} y_{n_{i}-1}\right)\right)\right| \leq f_{n_{i}}\left(Q T\left|K_{i}\left(x_{0} \wedge Q^{n_{i}-1} y_{n_{i}-1}\right)\right|\right) \\
& \quad \leq f_{n_{i}}\left(Q T R\left(x_{0} \wedge Q^{n_{i}-1} y_{n_{i}-1}\right)\right) \leq f_{n_{i}}\left(Q T R Q^{n_{i}-1} y_{n_{i}-1}\right) \\
& \leq f_{n_{i}}\left(Q^{n_{i}} T R y_{n_{i}-1}\right) \leq\left\|Q^{* n_{i}} f_{n_{i}}\right\| \cdot\|T R\| \cdot\left\|y_{n_{i}-1}\right\| \leq \frac{4\left\|x_{0}\right\|\|T R\|\left\|y_{n_{i}-1}\right\|}{\left\|y_{n_{i}}\right\|} \rightarrow 0 .
\end{aligned}
$$

Thus,

$$
f_{n_{i}}\left(Q T K_{i}\left(x_{0} \wedge Q^{n_{i}-1} y_{n_{i}-1}\right)\right) \rightarrow 0 .
$$

On the other hand,

$$
Q T K_{i}\left(x_{0} \wedge Q^{n_{i}-1} y_{n_{i}-1}\right) \rightarrow Q T w
$$

in norm. Since $f_{n_{i}} \stackrel{w^{*}}{\longrightarrow} g$, we conclude that $g(Q T w)=0$; hence $\left(Q^{*} g\right)(T w)=0$.

Since the ideal generated by Range $Q$ is dense and $g \neq 0$ is positive, we have $Q^{*} g \neq 0$. Let $Y$ be the linear span of $\langle Q] w$, that is, $Y=\operatorname{lin}\{T w: T \in\langle Q]\}$. Since $\langle Q]$ is a multiplicative semigroup, $Y$ is invariant under every $T \in\langle Q]$. It follows from $0 \neq w \in Y$ that $Y$ is non-zero. Finally, $\bar{Y} \neq X$ because $Q^{*} g$ vanishes on $Y$.

Suppose that $[0, R]$ localizes $B\left(x_{0}, 1\right) \cap\left[0, x_{0}\right]$ for some $x_{0} \geq 0$ and $\left\|x_{0}\right\|>1$. Then the vector $w$ constructed in the previous argument is positive. Let $E$ be the ideal generated by $\langle Q] w$, that is,

$$
E=\{y \in X:|y| \leq T w \text { for some } T \in\langle Q]\} .
$$

The ideal $E$ is non-trivial since $w \in E$, and it is easy to see that $E$ is invariant under $\langle Q]$. Since the positive functional $Q^{*} g$ vanishes on $T w$ it must also vanish on $E$; consequently $\bar{E} \neq X$ since $Q^{*} g \neq 0$.

Remark 4. It was shown in GT that with some minor adjustments, Theorem 2 can be extended from Banach lattices to ordered Banach spaces with generating cones. In a similar fashion, Theorem 3 can be extended to such spaces as well.

Next we present several applications of Theorem 3. Recall that an operator on a Banach lattice is $A M$-compact if it maps order bounded sets to relatively compact sets. In [FTT08], the authors proved the following extension of earlier results by R. Drnovšek (see AA02, Theorems 10.44 and 10.50]): if $Q$ is a quasinilpotent positive operator on a Banach lattice with a quasiinterior point such that some 
operator in $[Q\rangle$ dominates a non-zero $A M$-compact operator, then $[Q\rangle$ has an invariant closed ideal. Our next theorem provides a similar result for $\langle Q]$.

Theorem 5. If $Q$ is a positive quasinilpotent operator and there exists a nonzero AM-compact operator $K$ dominated by an operator in $\langle Q]$, then $\langle Q]$ has an invariant subspace. Furthermore, if $K \geq 0$, then $\langle Q]$ has a closed invariant ideal.

Proof. Let $K$ be a non-zero $A M$-compact operator dominated by an operator $R \in\langle Q]$. We can find $x_{0} \geq 0$ with $\left\|x_{0}\right\|>1$ such that $0 \notin \overline{K\left(B\left(x_{0}, 1\right) \cap\left[0, x_{0}\right]\right)}$. Therefore, the set of operators dominated by $R$ localizes $B\left(x_{0}, 1\right) \cap\left[0, x_{0}\right]$. Theorem 3 completes the proof.

Since every compact operator is an $A M$-compact operator, we have the following simple consequence of Theorem 5 .

Corollary 6. If $Q$ is a positive quasinilpotent operator and there exists a non-zero compact operator $K$ dominated by an operator in $\langle Q]$, then $\langle Q]$ has an invariant subspace. Furthermore, if $K \geq 0$, then $\langle Q]$ has a closed invariant ideal.

Following AA02 we give the following definition.

Definition 7. A positive operator $Q: X \rightarrow X$ is compact-friendly if there exist three operators $R, K$, and $C \neq 0$ such that $R Q=Q R, K$ is compact, and $C$ is dominated by both $R$ and $K$.

Remark 8. If $Q$ is a quasinilpotent compact-friendly operator and $C^{3} \neq 0$, where $C$ is as in Definition [7, then $\langle Q]$ has a common invariant subspace. Indeed, by Theorem 16.14 of AB85, $C^{3}$ is compact and $C^{3}$ is dominated by $R^{3}$ which is in $\langle Q]$. Then we use Corollary 6. Furthermore, if $C$ is positive, then by Theorem 3 and Corollary [6, $\langle Q]$ has a common invariant ideal.

In Theorems 10.55 and 10.57 of AA02 it was shown that under certain assumptions, the super right-commutant $[Q\rangle$ of a quasinilpotent compact-friendly operator $Q$ has an invariant subspace. The next two theorems show that under similar assumptions, $\langle Q]$ has an invariant subspace. The proofs are similar to the proofs of Theorems 10.55 and 10.57 in AA02, but we use Corollary [6] instead of Drnovšek's theorem as we deal with $\langle Q]$ instead of $[Q\rangle$.

Theorem 9. If $Q$ is a quasinilpotent compact-friendly operator, then at least one of the following is true:

(i) for each sequence $\left\{T_{n}\right\}$ in $\langle Q]$ there exists a non-trivial closed ideal that is invariant under $Q$ and each $T_{n}$, or

(ii) $\langle Q]$ has an invariant subspace.

Proof. Without loss of generality we can assume that $\|Q\|<1$ and suppose that $\left(T_{n}\right)$ is a sequence in $\langle Q]$. Pick arbitrary scalars $\alpha_{n}>0$ that are small enough so that the positive operator $T=\sum_{n=1}^{\infty} \alpha_{n} T_{n}$ exists and $\|Q+T\|<1$. Since $\langle Q]$ is a norm closed additive semigroup, it follows that the positive operator $A=$ $\sum_{n=0}^{\infty}(Q+T)^{n}$ belongs to $\langle Q]$.

For each $x>0$ we denote by $J_{x}$ the principal ideal generated by $A x$;

$$
J_{x}=\{y \in X:|y| \leq \lambda A x \text { for some } \lambda>0\} .
$$

It follows from $x \leq A x$ that $x \in J_{x}$, so that $J_{x} \neq 0$. 
Observe that $J_{x}$ is $(Q+T)$-invariant. Since $0 \leq Q, T \leq Q+T, J_{x}$ is invariant under $Q$ and $T$ and thus it is also $T_{n}$-invariant for each $n$. Therefore, if $\overline{J_{x}} \neq X$ for some $x>0$, then $\overline{J_{x}}$ is the desired invariant ideal.

Suppose $\overline{J_{x}}=X$ for each $x>0$. Then following the proof of Theorem 10.55 in AA02, we can construct a compact operator which is dominated by some $S \in\langle Q]$. Then Corollary [6 guarantees that $\langle Q]$ has an invariant subspace.

Remark 10. If $X$ is order complete, then we may assume that the operator $C$ in Definition 7 is positive. Indeed, take $x \geq 0$. For each $y \in[-x, x]$, we have $|C y| \leq K|y| \leq K x$. Then $|C| x=\sup _{y \in[-x, x]}|C y| \leq K x$, so that $|C| \leq K$. Likewise, $|C| \leq R$.

Theorem 11. If a non-zero compact-friendly operator $Q$ on an order complete Banach lattice is quasinilpotent, then $\langle Q]$ has a non-trivial closed invariant ideal.

Proof. For each $x>0$ we denote by $J_{x}$ the ideal generated by the orbit $\langle Q]$, that is,

$$
J_{x}=\{y \in X:|y| \leq T x \text { for some } T \in\langle Q]\} .
$$

Since $x \in J_{x}$, we have $J_{x} \neq 0$. Note that $J_{x}$ is invariant under each $T \in\langle Q]$. Therefore, if $\overline{J_{x}} \neq X$ for some $x>0$, then $\overline{J_{x}}$ is a $\langle Q]$-invariant closed ideal. So, suppose $\overline{J_{x}}=X$ for each $x>0$.

By Remark 10, there exist three positive non-zero operators $R, K$ and $C$ such that $R Q=Q R, C \leq R, C \leq K$, and $K$ is compact.

Claim: For every $x>0$, there exists $A \in\langle Q]$ such that $C A x>0$. Indeed, since $\overline{J_{x}}=X$ and $C \neq 0$, there exists a positive $y \in J_{x}$ such that $C y>0$. Then $y \leq A x$ for some $A \in\langle Q]$; hence $C A x>0$.

Fix any $x>0$. Applying the claim three times, we find $A_{1}, A_{2}, A_{3} \in\langle Q]$ such that $C A_{3} C A_{2} C A_{1} x>0$. Let $S=C A_{3} C A_{2} C A_{1}$. Then $S \neq 0$ and $C A_{i} \leq K A_{i}$ $(i=1,2,3)$; hence $S$ is compact by Theorem 16.14 of [AB85. Also, $0 \leq S \leq$ $R A_{3} R A_{2} R A_{1} \in\langle Q]$. Then Corollary 6 guarantees that $\langle Q]$ has a non-trivial closed invariant ideal.

The arguments in this paper are done for a real Banach lattice for simplicity. However, they work for complex Banach lattices with straightforward modifications.

\section{ACKNOWLEDGEMENT}

Special thanks are due to my advisor, Vladimir G. Troitsky, for suggesting the problems to me, helpful discussions, and reviewing the manuscript.

\section{REFERENCES}

[AA02] Y. A. Abramovich and C. D. Aliprantis, An Invitation to Operator Theory, Graduate Studies in Mathematics, vol. 50, American Mathematical Society, Providence, RI, 2002. MR.1921782(2003h:47072)

[AB85] C. D. Aliprantis and O. Burkinshaw, Positive Operators, Pure and Applied Mathematics, vol. 119, Academic Press Inc., Orlando, FL, 1985. MR809372 (87h:47086)

[AT05] R. Anisca and V. G. Troitsky, Minimal vectors of positive operators, Indiana Univ. Math. J., 54(3), 2005, 861-872. MR2151236 (2006c:47041)

[AE98] S. Ansari and P. Enflo, Extremal vectors and invariant subspaces, Trans. Amer. Math. Soc., 350, 1998, no. 2, 539-558. MR1407476 (98d:47019)

[D01] R. Drnovšek, Common invariant subspaces for collections of operators, Integral Eq. Oper. Th., 39, 2001, 253-266. MR1818060 (2001m:47012) 
[FTT08] J. Flores, P. Tradacete and V. G. Troitsky, Invariant subspaces of positive strictly singular operators on Banach lattices, J. Math. Anal. Appl., 343, 2008, 743-751.

[GT] H. Gessesse and V. G. Troitsky, Invariant subspaces of positive quasinilpotent operators on ordered Banach spaces, Positivity, 12, 2008, 193-208.

[Tr04] V. G. Troitsky, Minimal vectors in arbitrary Banach spaces, Proc. American Math. Soc., 132, 2004, 1177-1180. MR2045435 (2005a:47010)

Department of Mathematical and Statistical Sciences, University of Alberta, Edmonton, Alberta, T6G 2G1, Canada

E-mail address: hgessesse@math.ualberta.ca 